\title{
Fortschritte der Onkologie: mehr als neue, teure Medikamente
}

Markus Borner

Prof. Dr. med., Co-Präsident SGMO

Noch vor 20 Jahren hat man von neuen Therapieansätzen, welche die Onkologie revolutionieren, nur geträumt. Nun, da sie Wirklichkeit geworden sind, zeigen sich zwar beeindruckende Verbesserungen der Lebenszeit und -qualität, aber es stellen sich auch neue Fragen wie beispielsweise: Welche Patienten profitieren von den neuen Medikamenten? Und was, wenn die Zulassung nicht mehr mit der Entwicklung Schritt halten kann? Und, vor allem, wie können Patienten trotz Krebsdiagnose ein glückliches Leben führen?

Bevor eine Therapie überhaupt beginnt, müssen Ängste abgebaut und wichtige Inhalte vermittelt werden. Onkologen kümmern sich deshalb auch um die kommunikativen, psychoonkologischen und palliativen Be-

Ein Facharzt Onkologie muss neu eine palliativmedizinische Zusatzausbildung absolvieren.

dürfnisse ihrer Patienten und haben sich als die integrierende Spezialität für Tumorkranke etabliert in einer partnerschaftlichen Betreuungsgemeinschaft mit dem Hausarzt ${ }^{1}$ und dem Organspezialisten ${ }^{1}$.

\section{Verbesserte Behandlung mit ganzheit- lichem Blick}

Frau K., eine rüstige Witwe mit vier Grosskindern, sucht wegen Rückenschmerzen die Notfallstation auf, und es wird ein Brustkrebs mit Knochenmetastasen gefunden. Der Abteilungsarzt eröffnet, dass es sich um eine palliative Situation handle, und Frau K. verliert ihren Lebensmut, bis ihr Hausarzt sie einer Onkologin ${ }^{1} \mathrm{zu}-$ weist.

Eine Krebsdiagnose ist für viele Menschen das einschneidendste Ereignis in ihrem Leben. Das müsste es aber nicht sein, beträgt das 5-Jahres-Überleben mit «Krebs» doch weit über $50 \%$, und in vielen Fällen ist sogar eine Heilung möglich. Mit schuld an der Dramatik sind Begriffe wie «Krebs» und «Chemotherapie», die eigentlich in der Zeit der molekularen Medizin gar nicht mehr existieren dürften und gerade in ihrer ominösen Unschärfe enorm angstauslösend sind. Es gibt ja nicht «den Krebs», sondern mehrere hundert Krankheiten, denen gemeinsam ist, dass sich körpereigene Zellen unkontrolliert vermehren. In Abhängigkeit von der Genetik, dem betroffenen Organ und der Ausbreitung bei Diagnose unterscheiden sich Behandlungsmöglichkeiten und Prognose: Eine Heilung oder zumindest Kontrolle über lange Zeit ist durchaus - und immer häufiger - möglich. Auch «die Chemotherapie» gibt es nicht: Die in der Onkologie eingesetzten Medikamente sind nicht chemischer als Herzmedikamente oder Schmerzmittel - im Gegenteil: Das Paradigma der biologischen Therapie ist kaum in einem Gebiet der modernen Medizin besser anwendbar als in der Onkologie. Der Begriff "palliativ» wird ebenfalls unpräzis eingesetzt, sowohl für Tumortherapien zur Verbesserung und Verlängerung des Lebens als auch in der terminalen Krankheitssituation, wenn die Behandlung des Tumors selber nicht mehr sinnvoll scheint. Schon allein solche Erklärungen beim Erstgespräch entspannen die Betroffenen enorm. Nicht selten hört man den Satz: «Wenn ich das gewusst hätte, hätte ich nicht solche Angst gehabt.» Nicht von ungefähr sind auch kommunikative Kompetenzen ein wichtiger Teil der onkologischen Weiterbildung. Zudem muss ein Facharzt Onkologie neu eine palliativmedizinische Zusatzausbildung absolvieren. So kann er bei Patienten und ihren Familien die in diesen schwierigen Lebenssituationen notwendige Entscheidungskompetenz fördern, was nicht nur die Lebensqualität aller Beteiligten erhöht, sondern auch hilft, Kosten zu senken [1, 2].

\section{Mit Überzeugungsarbeit zum Therapie- erfolg}

Trotz den Durchbrüchen der zielgerichteten (molekularen) Tumortherapie basieren zurzeit noch alle kurati- 
Herr S., ein 25-jähriger Computerfachmann, erhält die Diagnose eines metastasierten Keimzelltumors und die Empfehlung einer kurativen Chemotherapie. Er hat von einer zielgerichteten und wenig toxischen Immuntherapie - allerdings beim Lungenkrebs gehört und ist enttäuscht, dass er nicht davon profitieren kann.

ven Ansätze auf der «altmodischen» Chemotherapie. Verschiedene Biomoleküle können zwar zusätzlich die Heilungschancen erhöhen, wie z.B. das Trastuzumab beim HER+-Brustkrebs oder das Rituximab bei der Behandlung von Lymphdrüsenkrebs. Die unkritische $\mathrm{Pu}$ blikation von medizinischen Inhalten in der Laienpresse löst jedoch oft eine unkritische Nachfrage aus, der dann eine grosse Ernüchterung folgt, wie aktuell in Bezug auf die medikamentöse Immuntherapie zu sehen ist. Herr S. konnte nur mit Mühe davon abgehalten werden, noch eine Zweitmeinung in Deutschland vier Wochen später abzuwarten - eine Verzögerung, die die Erfolgschancen seiner Behandlung unnötig reduziert hätte. Unter der Chemotherapie ist der Tumor verschwunden, Herrn S. geht es gut, und seine Heilungschancen liegen über $80 \%$. Dazu waren stundenlange Diskussionen und eine grosse Erfahrung mit komplexen Chemotherapien und deren Nebenwirkungen notwendig. Da die Facharztausbildung in der Onkologie nicht organspezifisch ist, gewährleistet sie eine hohe Expertise in Bezug auf die meist organübergreifend eingesetzten Tumormedikamente.

\section{Erfolge durch Anwendung noch nicht registrierter Medikamente}

Herr V., ein 59-jähriger Primarlehrer, wurde wegen eines metastasierten Harnblasenkarzinoms vor drei Jahren mit Chemotherapie behandelt. Nun wurde ein Rezidiv im Bauchraum festgestellt. Weil die verfügbaren Therapien allesamt nicht kassenpflichtig sind, organisiert die Onkologin über die Herstellerfirma eine Immuntherapie. Die Lebensqualität von Herrn V. normalisiert sich, eineTumoraktivität lässt sich seit elf Monaten nicht mehr feststellen. Herr V. arbeitet hochmotiviert weiter in seinem Beruf.

Da moderne Tumormedikamente in komplexe molekulare Netzwerke eingreifen, ist häufig nicht vorauszusehen, welche Patienten von diesen Therapieansätzen profitieren werden. Nur wenige Patienten sprechen an, dafür häufig sehr nachhaltig und meist bei guter Verträglichkeit. Die Durchführung grosser randomisierter Studien, wie von den Zulassungsbehörden gefordert, ist aus methodischen Gründen oft unmöglich. Die meist kleinen Zielgruppen werden als Rechtfertigung für die hohen Preise dieser Arzneien gebraucht, neue Vergütungsmodelle sind erst in Diskussion. Ein Onkologe muss einen zunehmenden Anteil seiner Arbeitszeit darauf verwenden, Patienten den Zugang zu noch nicht registrierten Medikamenten zu organisieren Entsprechend katastrophal wirkt sich eine Beschränkung der Vergütung dieser Zeit auf die Versorgung aus, zumal die Industrie in diesen Situationen häufig die

Die medikamentösen Therapiemöglichkeiten und die ganzheitliche Betreuung haben in der Onkologie grosse Fortschritte erzielt.

Medikamente gratis zur Verfügung stellen würde, so dass für das Gesundheitswesen Kosten gespart werden könnten. Ebenso sollte der Onkologe die Teilnahme an klinischen Studien fördern, da nur so die notwendige Grundlage für den Fortschritt geschaffen werden kann.

\section{Zusammenfassung}

Die medikamentösen Therapiemöglichkeiten und die ganzheitliche Betreuung haben in der Onkologie grosse Fortschritte erzielt. Auch bei den meist unheilbaren metastasierten Leiden hat sich die Dauer des Lebens verlängert und die Qualität verbessert. Die Zahl an molekularen Medikamenten nimmt rasch zu - nur wissen wir häufig noch nicht, wer ansprechen wird, und die Behandlung ist sehr teuer. Der Patient muss durch diese Unsicherheiten geführt werden, und sozialkompatible Lösungen müssen gesucht werden - das braucht Zeit. In den letzten 20 Jahren hat sich die Onkologie zu einer Disziplin entwickelt, die sich gesamtheitlich um die Bedürfnisse des Patienten kümmert und nicht nur um rein diagnostische und therapeutische Aspekte.

Die in diesem Artikel geäusserte Meinung muss sich nicht notwendigerweise mit der Meinung aller Mitglieder der Fachgesellschaft decken.

\section{Literatur}

1 Sher DJ. Economic Benefits of Palliative Care Consultation Continue to Unfold. J Clin Oncol. 2015;33(25):2723-4.

2 Borner M, Rauch D. Palliative Chemotherapie oder doch besser Palliative Care? Swiss Medical Forum. 2015;15(16):334-9. 